\title{
Modeling of Car-Following Required Safe Distance Based on Molecular Dynamics
}

\author{
Dayi Qu, Xiufeng Chen, Wansan Yang, and Xiaohua Bian \\ Automobile and Transportation College, Qingdao Technological University, Qingdao 266033, China \\ Correspondence should be addressed to Dayi Qu; dyqu@263.net
}

Received 1 October 2013; Revised 18 November 2013; Accepted 4 December 2013; Published 8 January 2014

Academic Editor: Wuhong Wang

Copyright ( 2014 Dayi Qu et al. This is an open access article distributed under the Creative Commons Attribution License, which permits unrestricted use, distribution, and reproduction in any medium, provided the original work is properly cited.

\begin{abstract}
In car-following procedure, some distances are reserved between the vehicles, through which drivers can avoid collisions with vehicles before and after them in the same lane and keep a reasonable clearance with lateral vehicles. This paper investigates characters of vehicle operating safety in car following state based on required safe distance. To tackle this problem, we probe into required safe distance and car-following model using molecular dynamics, covering longitudinal and lateral safe distance. The model was developed and implemented to describe the relationship between longitudinal safe distance and lateral safe distance under the condition where the leader keeps uniform deceleration. The results obtained herein are deemed valuable for car-following theory and microscopic traffic simulation.
\end{abstract}

\section{Introduction}

Car-following models elaborate the situation that vehicles follow one another in the same lane and capture drivers' maneuvering decisions under different conditions. The models are the most fundamental part of microscopic traffic simulation [1]. From the research of Reuschel and Pipes using the operational research theory method on car-following, the models can be classified as stimulus-response models (Gazis et al., 1961; Newell, 1961), safe distance models (Gipps, 1981), psychophysical models (Wiedemann, 1974), and artificial intelligence models (Kikuchi and Chakroborty, 1992; Wu et al., 2000) [2-6]. Among them, the car-following model based on the safe distance has broadly been used in practice [7].

Parker put forward an expectation distance model for the first time through the research on car-following behavior on the fast road sections [8]. Peter Hides carried out further research on microscopic behavior of urban traffic flow and preliminarily established car-following models for urban traffic flow based on expectation distance [9]. Zhang et al. gave deep research on psychological and physical reaction mechanism of the driver and presented multiregime model based on the driver's psychological reaction [10]. At present, the car-following model based on safe distance has been widely applied to microscopic simulation of road traffic, which is one of the hot research topics in the field of traffic engineering $[11,12]$. Reaction time for a car-following maneuver responding to an unexpected hazard in the roadway has been conducted in several studies [13-18]. From their results, the mean reaction times identified are rarely greater than 1.50 seconds. The safe distances in the articles above all gave the longitudinal clearance from a preceding vehicle in following procedure, while there always exists lateral impact in carfollowing. Gunay carried out research taking into lateral clearances for vehicles change and established the model taking the lateral positions of vehicles into account [19].

This paper concentrates on the model of car-following required safe distance. Required safe distance is necessary for ensuring the driving safety in car following procedure, while the factors such as the longitudinal clearances between vehicles and the speed also have implications on the vehicle. Thus, we make analysis on the influencing factors and perform the simulation for the required safe distance and lateral safe distance under different speed conditions of the leader and the follower, which indicates that the model of required safe distance both benefits the operating safety and driving efficiency.

The structure of the paper is organized as follows. After the introduction, analysis of molecular dynamics is described 
combined with the car following procedure in Section 2. Section 3 states the models of required safe distance including required safe distance and lateral safe distance. The influencing factors are illustrated from the perspective of longitudinal clearance and the speed in Section 4. Section 5 presents the test and validation to demonstrate the application of the model. The final section summarizes the findings and the conclusions of the paper.

\section{Analysis of Molecular Dynamics}

Among the molecules, attractive force and repulsive force simultaneously exist, both of which increase with the distance decreasing. But their changing rules are different that the attractive force declines more slowly. Molecular forces makes the molecules difficult to approach and be far away, as a phenomenon that the gas is difficult to be compressed and be expanded at a certain temperature. Vehicles in the carfollowing fleet show similar characteristics. From the aspect of safety, drivers try to keep a safe distance with the leader; at a certain speed, the phenomenon shows that the fleet is difficult to be compressed. So if the leader speed increases, for the sake of the efficiency, the follower will accelerate not to be fallen behind by the leader for long time. Thus, the clearance between adjacent vehicles will not be continually enlarged and the whole fleet will not be expended too much. Here, we define these characters as molecular car-following behavior, the theory of studying which is called molecular car-following theory.

In the car-following procedure, each vehicle is independent, but it also affects one another. Relative to the leader, every vehicle is the follower, while it is the leader that is relative to the rear vehicle. Similar to the dynamics relationship of molecules, there is an equilibrium distance called required safe distance in the fleet, just like the clearance between molecules where the resultant force is zero. As shown in Figure 1, on the assumption that the braking efficiency of three vehicles is identical, the braking distance is only influenced by the speed. And the required safe distance is proposed into front, lateral, and rear distance according to different directions. We call the furthest distance the follower keeps with the leader as required frontier and the furthest distance the follower keeps with the rear vehicle as required trailing edge. With respect to a vehicle, required frontier is actively obtained, while required trailing edge is passively obtained.

We define the state that the follower positions at the required trailing edge and drives at the same speed of the leader as equilibrium state. As illustrated in Figure 1, the front required safe distance is equal to the equilibrium distance in molecular dynamics with the same direction as vehicles' and also brings about influence on the followers. With speed as reference to the leader itself, the minimum safe distance the leader expects the follower to keep is called rear distance. Moreover, for the purpose to avoid confusing with the lateral safe distance, the front safe distance and the rear distance are together defined as required safe distance.

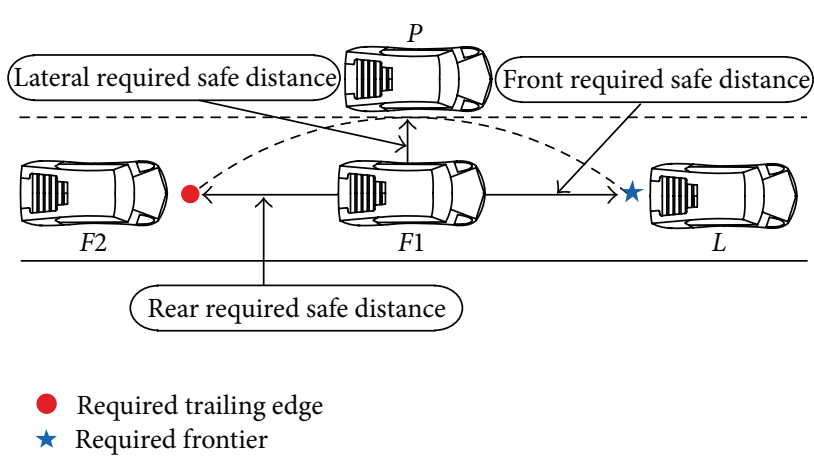

FIGURE 1: Vehicle required safe distance in car-following.

When the driver perceives the leader's travelling state being changed, the required safe distance is the minimum safe distance that the driver starts to act until stopping braking. The follower will not be fallen behind for long time considering the efficiency while will not be too near considering the safety in the following procedure.

\section{Modeling Required Safe Distance}

3.1. Required Safe Distance. When the leader maintains the braking state of uniform deceleration, supposing that $v_{L}$, and $v_{F}$ are respectively the original speeds of the leader and the follower and $a_{L m}$, and $a_{F m}$ are, respectively, the maximum decelerations, then the relative speed is $\left(v_{F}-v_{L}\right)$.

The finding shows that $t_{r}$ is the sum of reaction time and braking coordination time ranging from 0.8 second to 1.0 second; $t_{i}$ is the build-up time of deceleration ranging from 0.1 second to 0.2 second; then the braking distance can be get

$$
X=v_{0}\left(t_{r}+\frac{t_{i}}{2}\right)+\frac{v_{0}^{2}}{2 a_{m}}
$$

where $v_{0}$ is the initial speed, $a_{m}$ is the maximum deceleration.

If the leader operates with uniform motion or uniform acceleration, the follower can easily keep following in a safe state. After a period of time, the follower will drive at its original speed not to collide with the leader as long as the leader keeps a uniform motion. The follower always tries to achieve speed close to the leader's to improve travelling efficiency. If the leader conducts uniform deceleration, the required safe distance of the follower should be analyzed based on the changes of vehicles' speed before and after.

(1) $v_{F}>v_{L}$. Under the condition that the speed of the follower is faster than the leader and the leader does a uniform deceleration, the follower may collide with the leader without timely deceleration. At this moment, the leader will actively do uniform deceleration and the braking distance with no reaction time is

$$
X_{L}=\frac{v_{L}^{2}+v_{L} t_{i} a_{L m}}{2 a_{L m}}
$$


If the follower decelerates after the time $t_{r}$, where $t_{r}$ is the perceiving time after the leader changed, then the braking distance is

$$
X_{F}=v_{F} t_{r}+\frac{v_{F}^{2}+v_{F} t_{i} a_{F m}}{2 a_{F m}} .
$$

From the analysis above, we can get required safe distance of the follower in uniform deceleration condition as

$$
X_{R}=v_{F} t_{r}+\frac{v_{d} t_{i}}{2}+\frac{v_{F}^{2}}{2 a_{F m}}-\frac{v_{L}^{2}}{2 a_{L m}}+d,
$$

where $d$ is the minimum clearance between adjacent vehicles after stopping, ranging from 2 meters to 5 meters.

(2) $v_{F}=v_{L}$. This situation is similar to the above. So the required safe distance is:

$$
X_{R}=v_{F} t_{r}+\frac{v_{L}^{2}}{2}\left(\frac{1}{a_{F m}}-\frac{1}{a_{L m}}\right)+d .
$$

(3) $v_{F}<v_{L}$. If there is a bigger clearance than required safe distance between adjacent vehicles, two vehicles can keep a safe state during a period of time. But if the follower continues driving at the initial speed, collisions may occur later on. So the follower should decelerate when the speed of the follower approaches the leader speed.

Supposing that the leader decelerates with maximum deceleration when the speed of follower is equal to the leader speed, then the time from decelerate till this moment is

$$
t_{1}=t_{i}+\frac{\left(v_{L}-\left(a_{L m} t_{i} / 2\right)-v_{F}\right)}{a_{L m}} .
$$

During this time, the follower operates with the same speed, and the distance is

$$
X_{F 1}=v_{F} t_{i}+\frac{v_{F}}{a_{L m}}\left(v_{L}-\frac{a_{L m} t_{i}}{2}-v_{F}\right) .
$$

And when the follower must decelerate, the braking distance is:

$$
X_{F 2}=v_{F} t_{r}+\frac{v_{F}^{2}+v_{F} t_{i} a_{F m}}{2 a_{F m}} .
$$

So the total distance of the follower is

$$
X_{F}=v_{F}\left(t_{i}+t_{r}\right)-\frac{v_{F} v_{d}}{a_{L m}}+\frac{v_{F}^{2}}{2 a_{L m}} .
$$

And the leader keeps uniform deceleration and its driving distance is

$$
X_{L}=\frac{v_{L}^{2}+v_{L} t_{i} a_{L m}}{2 a_{L m}} .
$$

So required safe distance of the follower can be gotten as follows:

$$
X_{R}=v_{F}\left(t_{i}+t_{r}\right)-\frac{2 v_{F} v_{d}+v_{L}^{2}}{2 a_{L m}}+\frac{v_{F}^{2}}{2 a_{F m}}-\frac{v_{L} t_{i}}{2}+d .
$$

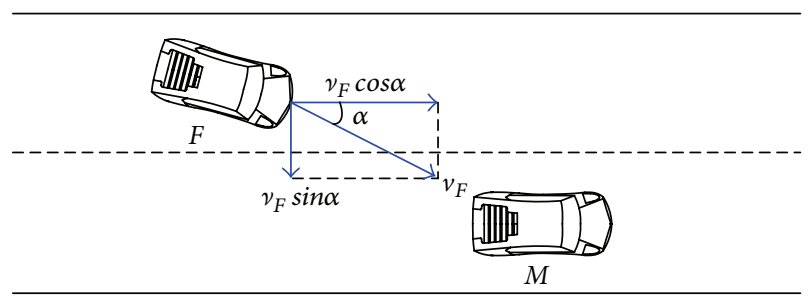

FIGURE 2: Speed decomposition diagram when the vehicle $F$ shifts.

3.2. Lateral Required Distance. The lateral required distance is the minimum lateral clearance that the vehicle keeps not to collide with the nearest one on the adjacent lane. Considering drivers' physical and mental characters, even if the lateral clearance is less than the lateral required distance demanded at a certain time, it does not necessarily cause accidents.

As illustrated in Figure 2, $\alpha$ is the angle at which the follower deviates from the target lane. If a large longitudinal clearance exists between the adjacent vehicles, the vehicle $F$ can adjust timely; then the vehicle $M$ will not result in heavy impact on the vehicle $F$. Moreover, the faster the speed of the follower is, the further the vehicle deviates during the reaction time and the more probably two vehicles collide. Thus the lateral required distance is influenced by both the longitudinal clearance and the vehicle speed.

At a certain time, $v_{F}$ represents the speed of the vehicle $F$ and $\alpha$ is the maximum deviation angle. In Figure 2, decomposing $v_{F}$ into lateral and longitudinal directions, $v_{F} \sin \alpha$ is the lateral velocity component and $v_{F} \cos \alpha$ is the longitudinal velocity component. At the end of the reaction time $t$, the lateral displacement component may be less than the lateral clearances, and it may lead to accidents. The lateral required distance can be written as

$$
X_{S}=v_{F} \cdot t \cdot \sin \alpha,
$$

where $t$ is the reaction time, $\alpha$ is the maximum possible deviation angle.

The analysis above reveals that the lateral required distance is linear with the speed of the follower.

Putting formulas (4) and (12) together, when $v_{F}>v_{L}$, the relationship between $X_{R}$ and $X_{S}$ is shown as follows:

$$
X_{R}=\frac{X_{S}}{t \cdot \sin \alpha} t_{r}+\frac{v_{d} t_{i}}{2}+\frac{X_{S}^{2}}{2 a_{F m} \cdot(t \cdot \sin \alpha)^{2}}-\frac{v_{L}^{2}}{2 a_{L m}}+d .
$$

Since formulas (4) and (12) are both monotonically increasing functions of the speed $v_{F}$, the lateral required distance $X_{S}$ increases with $X_{R}$ increasing.

Similarly, for $v_{F}=v_{L}$, the relationship between $X_{R}$ and $X_{S}$ is expressed as

$$
X_{R}=\frac{X_{S}}{t \cdot \sin \alpha} t_{r}+\frac{v_{L}^{2}}{2}\left(\frac{1}{a_{F m}}-\frac{1}{a_{L m}}\right)+d
$$




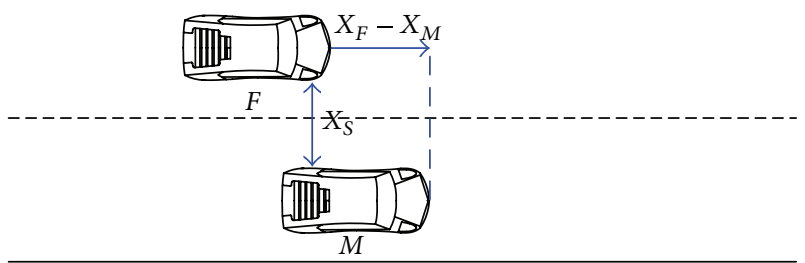

FIGURE 3: Positional relationship diagram between adjacent lane vehicles.

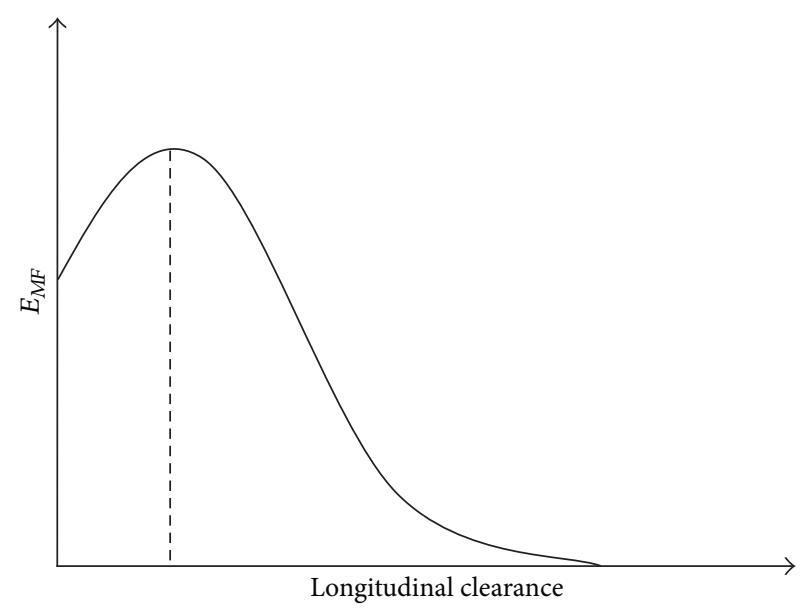

FIGURE 4: Influencing curve on follower of longitudinal clearance.

For $v_{F}<v_{L}$, the relationship between $X_{R}$ and $X_{S}$ is

$$
\begin{aligned}
X_{R}= & \frac{X_{S}}{t \cdot \sin \alpha}\left(t_{i}+t_{r}\right)-\frac{X_{S}}{t \cdot \sin \alpha} \cdot \frac{v_{d}}{a_{L m}}-\frac{v_{L}^{2}}{2 a_{L m}} \\
& +\left(\frac{X_{S}}{t \cdot \sin \alpha}\right)^{2} \cdot \frac{1}{2 a_{F m}}-\frac{v_{L} t_{i}}{2}+d .
\end{aligned}
$$

However, in the real-world situation, the lateral clearance between the follower and lateral vehicle should be a little greater than that defined above because the lateral clearance will be enlarged in some cases where the follower decelerates or avoids collisions laterally.

\section{Influencing Analysis of Influential Factors}

4.1. Influencing Analysis of Longitudinal Clearance. Figure 3 demonstrates that the longitudinal clearance $X_{F}-X_{M}$ between the two vehicles has influence on the follower in adjacent lane. The influencing curve is illustrated in Figure 4.

On the assumption that the longitudinal clearance is $\left(v_{F}\right.$. $\left.\cos \alpha-v_{M}\right) \cdot t$, it is exactly zero at the end of the reaction time, which is the most probable point where two vehicles collide. The study shows that under the condition where the longitudinal clearance and its speeds are unchanged, the influence on the follower by $X_{F}-X_{M}$ follows normal
TABLE 1: Table of safe distance model formula.

\begin{tabular}{lc}
\hline Model & Formula \\
\hline $\begin{array}{l}\text { Safe distance model based } \\
\text { on headway }\end{array}$ & $s_{1}=v_{0} t_{d}+d$ \\
$\begin{array}{l}\text { Safe distance model by } \\
\text { braking process }\end{array}$ & $s_{2}=v_{0} t_{d}+\frac{v_{0}^{2}}{2 a_{m}}+d$ \\
$\begin{array}{l}\text { The model of required safe } \\
\text { distance }\end{array}$ & $\begin{array}{c}\text { Models of required safe distance at } \\
\text { three following states as above } \\
\text { formulas (4), (5), and (11). }\end{array}$ \\
\hline
\end{tabular}

distribution. $E_{M F}$ is the influence on the follower by the lateral vehicle then

$$
E_{M F}=\frac{1}{\sqrt{2 \pi \sigma}} e^{-\left[X-\left(v_{F} \cdot \cos \alpha-v_{M}\right) \cdot t\right]^{2} / 2 \sigma^{2}}, \quad 0 \leq X<+\infty,
$$

where $X$ is the longitudinal clearance, $\sigma$ is a undetermined parameter. And at the point that the longitudinal clearance is $\left(v_{F} \cdot \cos \alpha-v_{M}\right) \cdot t$, the biggest influencing point is $1 / \sqrt{2 \pi \sigma}$.

4.2. Influencing Analysis of the Speed. In the driving procedure, the faster the speed is, the lighter steering wheel is for the driver, and the vehicle is more probably to produce the lateral deviation, which may directly put much mental pressure on drivers. In this case, the driver of the follower will drive away from the lateral vehicle or decelerate to avoid colliding.

As the lateral vehicle quickly approaches the follower, the following driver will concentrate on it. To a certain extent, the driver's pressure will increase. If the follower is within the range influenced by the lateral vehicle, the lateral clearance and the longitudinal clearance are unchanged, and the larger the speed difference between adjacent vehicles is, the stronger influence the lateral vehicle has on the follower; thus, drivers should decelerate to avoid dangers.

\section{Testing and Validation of the Models}

5.1. Simulation on the Required Safe Distance. Through the tests of three following states for $v_{F}>v_{L}, v_{F}=v_{L}$ and $v_{F}<$ $v_{L}$ the model of required safe distance and two traditional models are compared to analyze the difference. One of the two traditional models is based on headway and another is based on braking process. Formulas of the three models are shown in Table 1.

In order to make a better simulation and analysis compared with the traditional models of safe distance, we assign values to parameters of the formulas, as shown in Table 2.

The relationship between the safe distance and $v_{F}$ is, respectively, simulated as in Figure $5\left(v_{F}>v_{L}\right)$ and Figure 6 $\left(v_{F}=v_{L}\right)$ on the assumption of $v_{L}=50 \mathrm{~km} \cdot \mathrm{h}^{-1}$, and Figure 7 describes the relationship between the safe distance and $v_{L}$ supposing $v_{F}=50 \mathrm{~km} \cdot \mathrm{h}^{-1}$. From Figure 5, the result shows that the safe distance based on braking process is too large to decrease the road capacity, and the safe distance based on headway is too small to cause collision in car following procedure. While the model of required safe distance takes the driving characters of vehicles before and 
TABLE 2: Calibration table of formula parameters.

\begin{tabular}{lccccc}
\hline Parameter & $d(\mathrm{~m})$ & $t_{d}(\mathrm{~s})$ & $a_{m}\left(\mathrm{~m} \cdot \mathrm{s}^{-2}\right)$ & $t_{i}(\mathrm{~s})$ & $t_{r}(\mathrm{~s})$ \\
\hline Value & 4 & 1.6 & 7 & 0.2 & 0.9
\end{tabular}

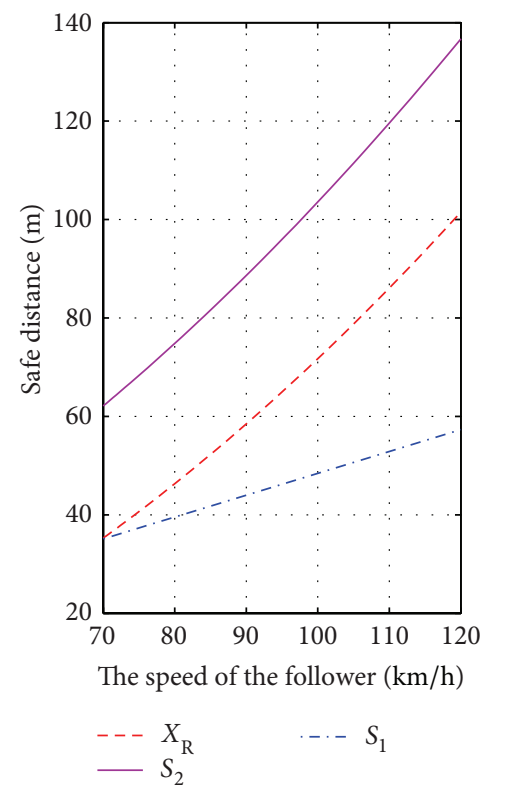

FIGURE 5: Simulation curve for $v_{F}>v_{L}$.

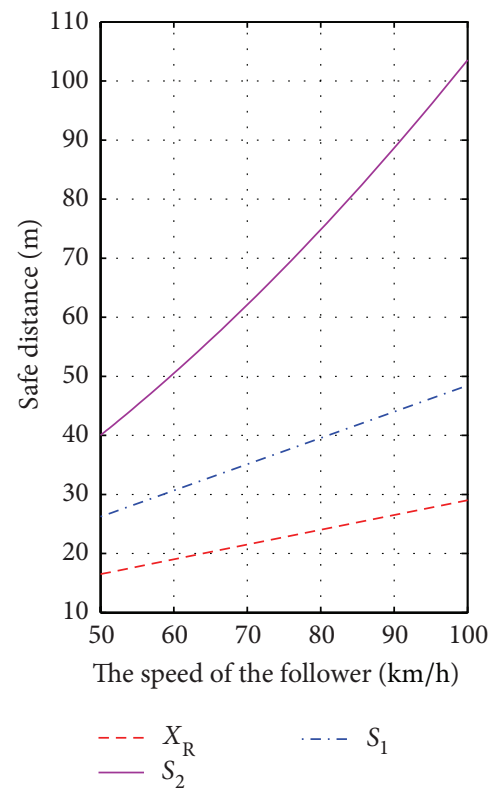

Figure 6: Simulation curve for $v_{F}=v_{L}$.

after into consideration, so the follower need not adjust the clearance when keeping the state consistent with the leader, which consequently avoids the situation above. Figures 6 and 7 demonstrates that safe distances based on two traditional models are larger, which ensures two vehicles not to collide but reduces travel efficiency. However, the model of required

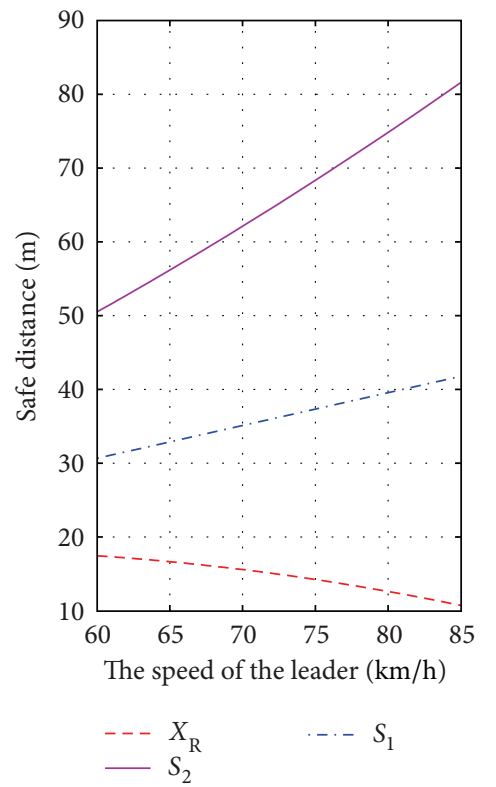

FIgURE 7: Simulation curve for $v_{F}<v_{L}$.

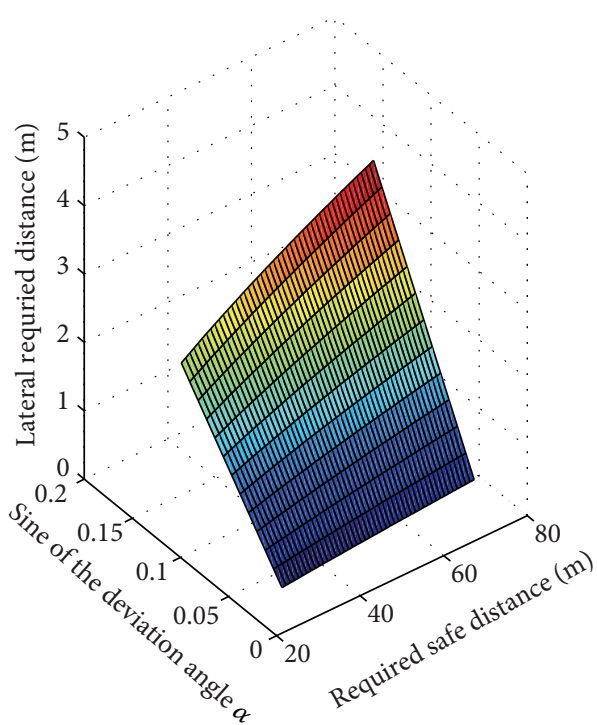

FIGURE 8: Simulation diagram for $v_{F}>v_{L}$.

safe distance not only ensures safe operation of vehicles but also improves travel efficiency.

5.2. Simulation on the Lateral Safe Distance. The simulation curves of the lateral required distance for $v_{F}>v_{L}, v_{F}=v_{L}$, and $v_{F}<v_{L}$ are illustrated in Figures 8, 9, and 10. Figures 8 and 9 are under the condition that the leader's speed is 


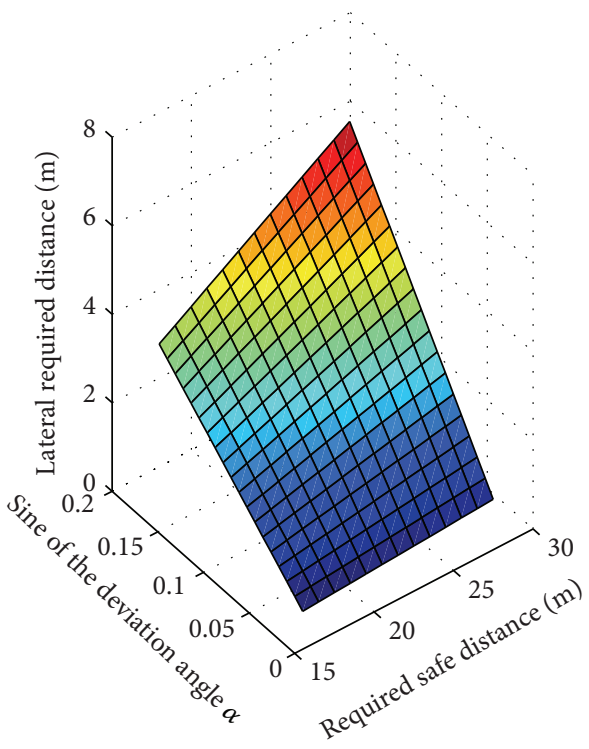

FIgURE 9: Simulation diagram for $v_{F}=v_{L}$.

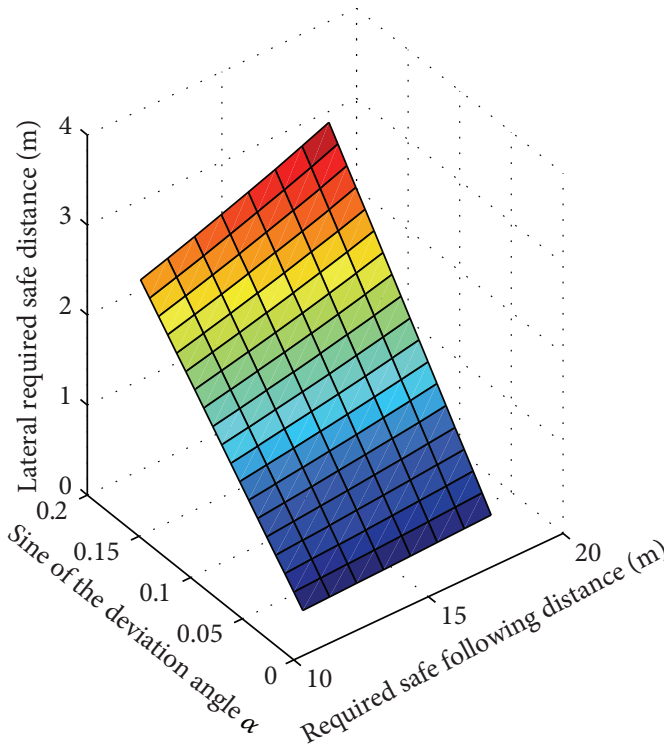

FIGURE 10: Simulation diagram for $v_{F}<v_{L}$.

$50 \mathrm{~km} \cdot \mathrm{h}^{-1}$, and Figure 10 is assuming that the leader's speed is $70 \mathrm{~km} \cdot \mathrm{h}^{-1}$. As shown in the figures, the lateral required distance increases with the deviation angle increasing, and with $X_{R}$ increasing, the changing amplitude will increase; similarly, the required safe distance also increases with $X_{R}$ increasing. But it almost has no impact on $X_{S}$ when $\alpha$ is small. If the angle $\alpha$ is large, this needs a larger lateral required distance changing largely with $v_{F}$ increase, and this is also consistent with the actual situation.

\section{Conclusion}

This paper presents the concept of car-following required safe distance, which is based on molecular dynamics. We develop the models of required safe distance and lateral safe distance and apply mathematical reasoning method to get their expressions. And the influence on lateral required distance is analyzed from the aspects of longitudinal clearance and the speed. For better understanding, we make the comparison between the model of required safe distance and two traditional models, which reveals that the model of required safe distance shows the priority in driving safety and efficiency. Finally, upon the completion of the tests and validation using the actual survey data in crossings, it is pointed out that the model of car-following required safe distance based on molecular dynamics is significant for vehicles driving safely and efficiently, which can provide effective theoretical basis for the adaptive cruise control system.

\section{Conflict of Interests}

The authors declare that there is no conflict of interests regarding the publication of this article.

\section{Acknowledgments}

The paper is supported by the National Natural Science Foundation of China (51178231) and Provincial Natural Science Foundation of Shandong (ZR2012EEL128). The authors would like to thank the referees.

\section{References}

[1] T. Toledo, "Driving behaviour: models and challenges," Transport Reviews, vol. 27, no. 1, pp. 65-84, 2007.

[2] D. C. Gazis, R. Herman, and R. W. Rothery, "Nonlinear followthe-leader models of traffic flow," Operation Research, vol. 9, no. 4, pp. 545-567, 1961.

[3] G. F. Newell, "Nonlinear effects in the dynamics of car following," Operation Research, vol. 9, no. 2, pp. 209-229, 1961.

[4] P. G. Gipps, "Behavioral car-following model for computer simulation," Transportation Research B, vol. 15, no. 2, pp. 105111, 1981.

[5] R. Wiedemann, Simulation of Road Traffic in Traffic Flow, University of Karlsruhe, Karlsruhe, Germany, 1974.

[6] C. Kikuchi and P. Chakroborty, "Car following model based on a fuzzy inference system," Transportation Research Record, vol. 1194, pp. 82-91, 1992.

[7] M. F. Aycin and R. F. Benekoha, "Comparison of car-following models for simulation," in Proceedings of the 78th Annual Meeting of Transportation Research Board (TRB '99), 1999.

[8] M. T. Parker, "The effect of heavy goods vehicles and following behaviour on capacity at motorway roadwork sites," Traffic Engineering \& Control, vol. 37, no. 9, pp. 524-531, 1996.

[9] H. Peter, "A car-following model for urban traffic simulation," Traffic Engineering \& Control, vol. 39, no. 5, pp. 300-302, 1998.

[10] Y. L. Zhang, J. E. Clark, and E. C. James, "A multivegime approach for microscopic traffic simulation," in Proceedings of the 77th Annual Meeting of Transportation Research Board (TRB '98), Washington, DC, USA, 1998.

[11] S. Druitt, "An introduction to microsimulation," Traffic Engineering \& Control, vol. 39, no. 9, pp. 480-483, 1998.

[12] E. R. Boer, "Car following from the driver's perspective," Transportation Research F, vol. 2, no. 4, pp. 201-206, 1999. 
[13] D. B. Fambro, R. J. Koppa, D. L. Picha, and K. Fitzpatrick, "Driver perception-brake response in stopping sight distance situations," Journal of the Transportation Research Board, vol. 1628, pp. 1-7, 1998.

[14] K. I. Ahmed, Modeling driver's acceleration and lane changing behaviors [Ph.D. thesis], Department of Civil and Environmental Engineering, Massachusetts Institute of Technology, 1999.

[15] G. S. Gurusinghe, T. Nakatsuji, Y. Tanaboriboon, K. Takahashi, and J. Suzuki, "A car-following model incorporating excess critical speed concept," Journal of Eastern Asia Society for Transportation Studies, vol. 4, no. 2, pp. 171-183, 2001.

[16] P. Ranjitkar, T. Nakatsuji, G. Gurusinghe, and Y. Azuta, "Carfollowing experiments using RTK GPS and stability characteristics of followers in platoon," in Proceedings of the 7th International Conference on Applications of Advanced Technology in Transportation, pp. 608-615, Boston, Mass, USA, 2002.

[17] Y. Uchiyama, K. Ebe, A. Kozato, T. Okada, and N. Sadato, “The neural substrates of driving at a safe distance: a functional MRI study," Neuroscience Letters, vol. 352, no. 3, pp. 199-202, 2003.

[18] J. S. Kong, F. Guo, and X. P. Wang, "A vehicle rear-end anticollision method base on safety distance model," Automotive Electronics, vol. 24, no. 11, pp. 251-253, 2008.

[19] B. Gunay, "Car following theory with lateral discomfort," Transportation Research B, vol. 41, no. 7, pp. 722-735, 2007. 


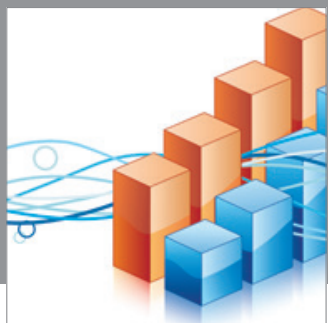

Advances in

Operations Research

mansans

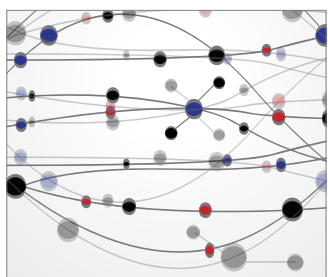

The Scientific World Journal
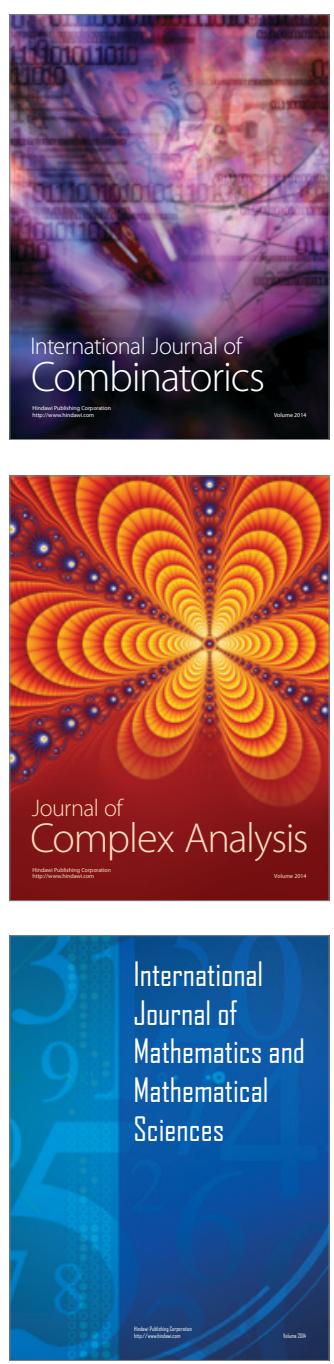
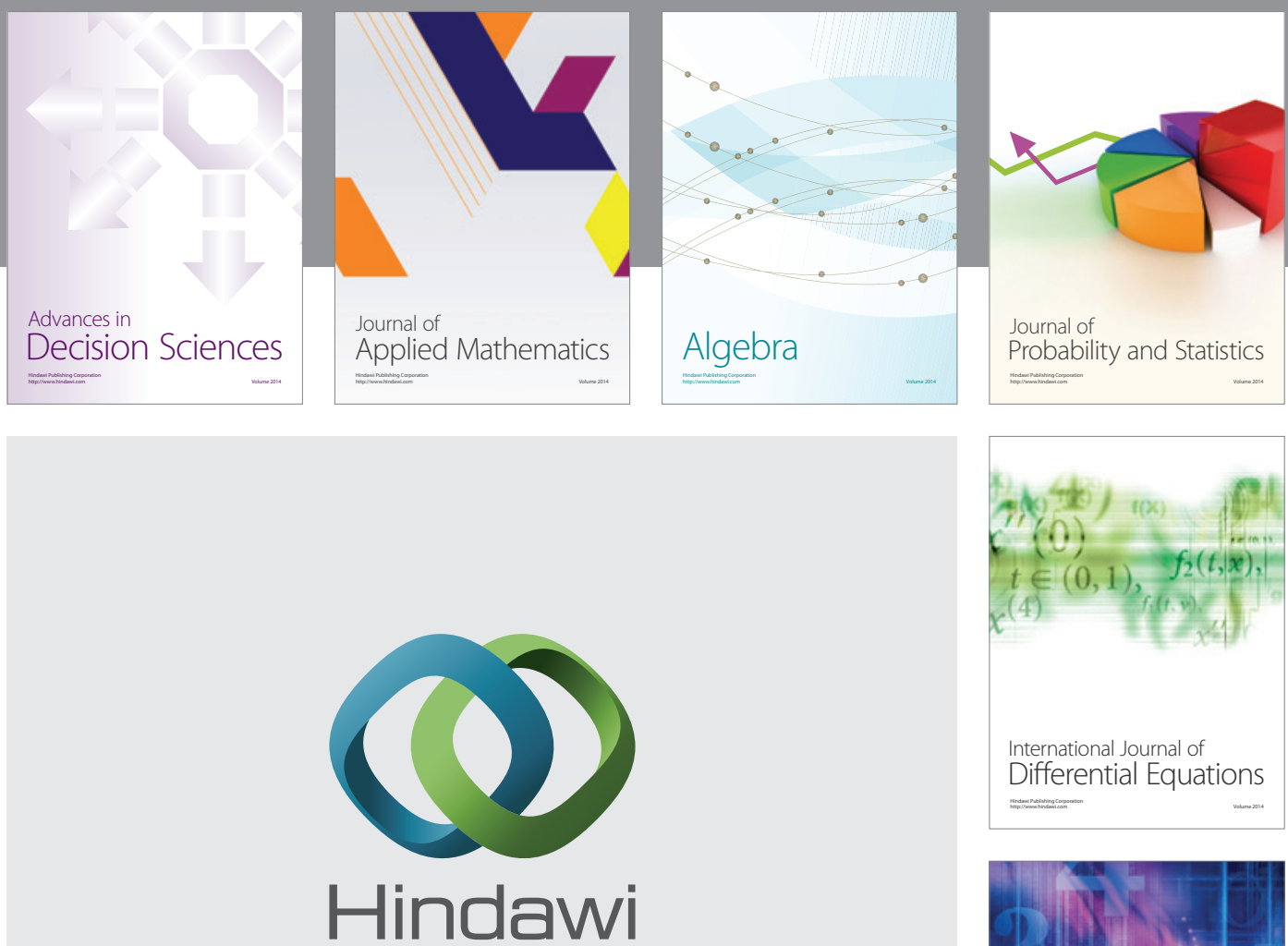

Submit your manuscripts at http://www.hindawi.com
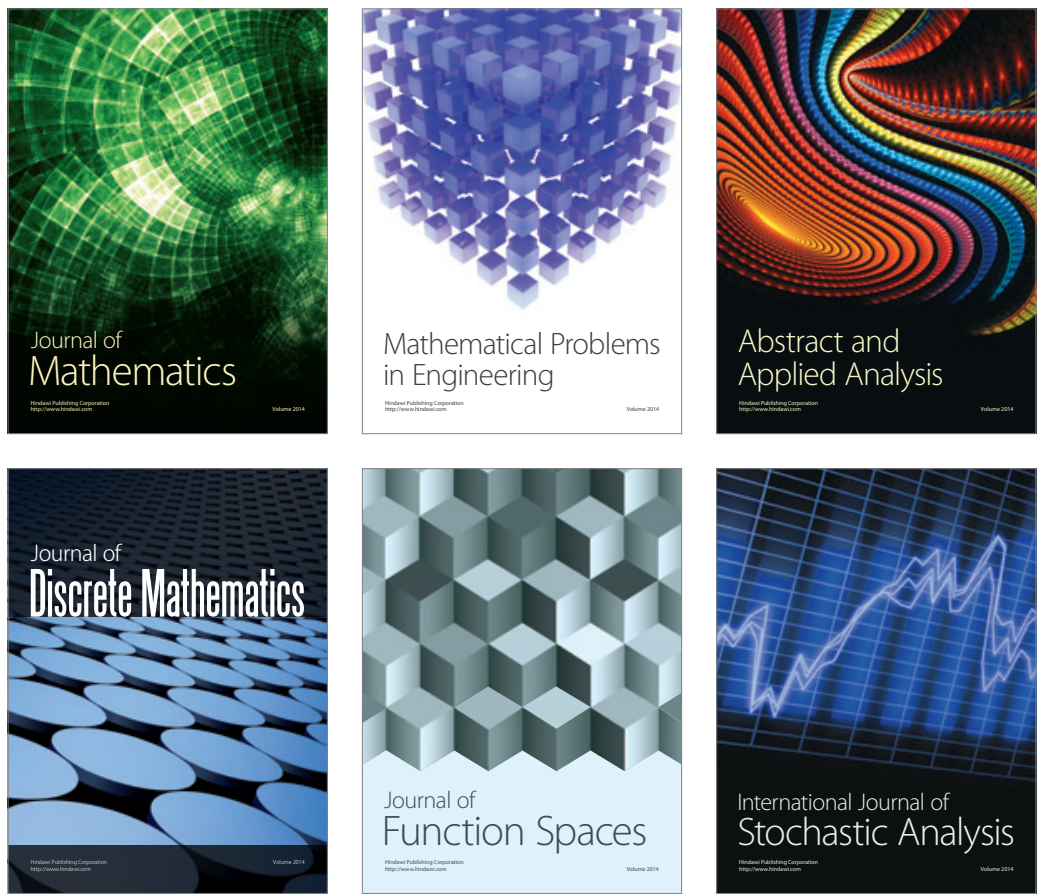

Journal of

Function Spaces

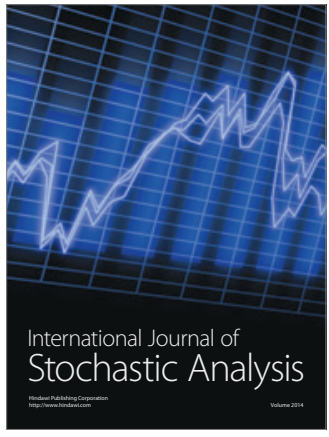

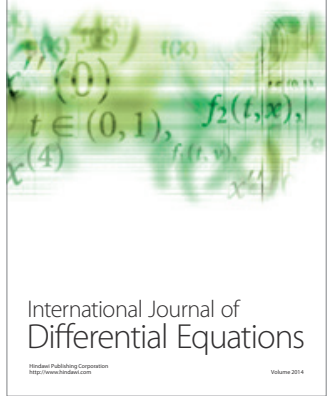
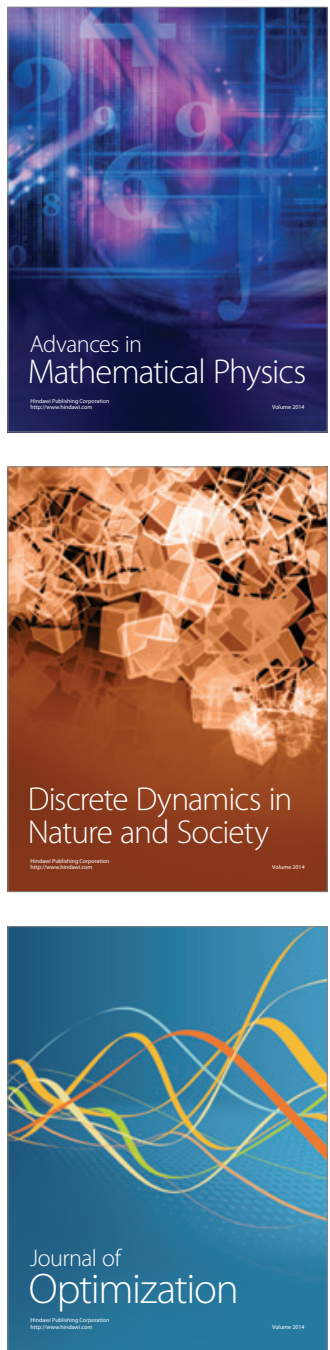